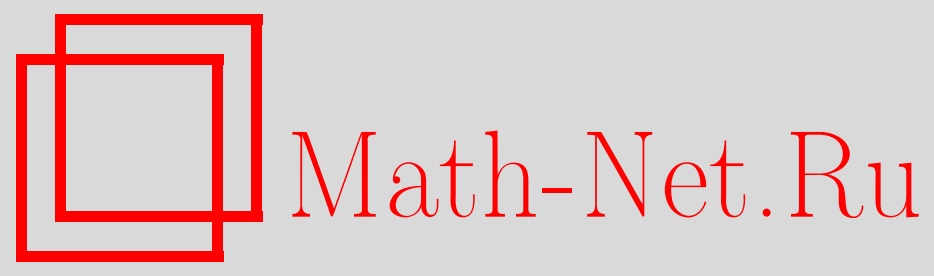

Э. Э. Гасанов, Нижняя оценка сложности включающего поиска в классе древовидных схем, Дискрет. матем., 1998, том 10, выпуск 1, 63-72

DOI: https://doi.org/10.4213/dm412

Использование Общероссийского математического портала Math-Net.Ru подразумевает, что вы прочитали и согласны с пользовательским соглашением http://www . mathnet.ru/rus/agreement

Параметры загрузки:

IP: 54.198 .67 .100

26 апреля 2023 г., 13:54:34

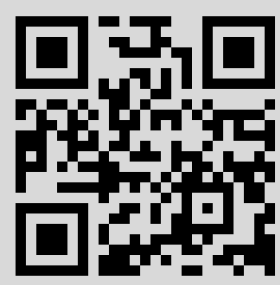




\title{
Нижняя оценка сложности включающего поиска в классе древовидных схем
}

\author{
(C) 1998 г. Э. Э. Гасанов
}

\begin{abstract}
В классе древовидных информационных сетей с базовым множеством переменных приведены примеры таких задач включающего поиска, что нижняя оценка их сложности по порядку больше среднего времени перечисления ответа.

Работа выполнена при поддержке Российского фонда фундаментальных исследований, проект 95-01-00597.
\end{abstract}

\section{1. Введение}

Известным фактом в теории информационного поиска является так называемая тривиальная нижняя оценка [1], которая говорит, что временная сложность задач поиска не меньше, чем время, необходимое на перечисление ответа. Для задачи включающего поиска, которая состоит в поиске в некотором конечном подмножестве булева куба, тех точек, которые находятся в отношении "не меньше покомпонентно" с точкой-запросом, в работе [2] получена нижняя оценка сложности, в два раза превышающая тривиальную нижнюю оценку. В данной работе найдены такие задачи включающего поиска, для которых в классе древовидных информационных сетей в случае, когда базовое множество есть множество переменных, получена нижняя оценка, по сравнению с которой тривиальная нижняя оценка меньше по порядку. Этот результат также справедлив в классе так называемых бесповторных информационных сетей, для которых в [3] доказана древовидность оптимальных сетей.

Автор выражает благодарность В. Б. Кудрявцеву и А. С. Подколзину за поддержку в работе.

\section{2. Основные понятия и формулировка результата}

Мы будем использовать терминологию и обозначения статьи [4]. Напомним понятие информационной сети с переключателями (ИСП) и другие необходимые понятия из [4].

Пусть $X$ - множество запросов, причем над $X$ опредєлено вероятностное пространство $\langle X, \sigma, \mathbf{P}\rangle$, где $\sigma$ - алгебра подмножеств множества $X, \mathbf{P}$ - вероятностная мера на $\sigma ; Y$ - множество записей (объектов поиска); $\rho$ - бинарное отношение на $X \times Y$, называемое отношением поиска; тройку $S=\langle X, Y, \rho\rangle$ (или пятерку 
$S=\langle X, Y, \rho, \sigma, \mathbf{P}\rangle$, если мы хотим подчеркнуть, какое именно вероятностное пространство над $X$ используется) будем называть типом или иногда более развернуто типом задач информационного поиска; тройку $I=\langle X, V, \rho\rangle$, где $V$ - некоторое конечное подмножество множества $Y$, в дальнейшем называемое библиотекой, будем называть задачей информационного поиска (ЗИП) типа $S=\langle X, Y, \rho\rangle$ (или ЗИП, принадлежащей типу $S$, и писать $I \in S$, обозначая эту принадлежность), и будем считать, что задача $I=\langle X, V, \rho\rangle$ состоит в перечислении для произвольно взятого запроса $x \in X$ всех тех и только тех записей из $V$, которые находятся в отношении $\rho$ с запросом $x$, то есть удовлетворяют запросу $x ; O(y, \rho)=\{x \in X: x \rho y\}$ - теиь записи $y \in Y$;

$$
N_{f}=\{x \in X: f(x)=1\},
$$

где $f$ - одноместный предикат, определенный на $X$, то есть $f: X \rightarrow\{0,1\}$; функция $\chi_{y, \rho}: X \rightarrow\{0,1\}$ такая, что $N_{\chi_{y, \rho}}=O(y, \rho)-$ характеристическая функция записи $y ; F$ - множество символов одноместных предикатов, определенных на множестве $X$, называемое базовым множеством предикатов; $G$ - множество символов одноместных переключателей, определенных на множестве $X$. Под переключателями будем понимать функции, областью значений которых являются конечные подмножества натурального ряда.

Пару $\mathscr{F}=\langle F, G\rangle$ назовем базовым множеством.

Если $n$ - натуральное число, а $g(x)$ - некий переключатель, то через $\xi_{g}^{n}(x)$ обозначим предикат, определенный на $X$, такой, что

$$
N_{\xi_{g}^{n}}=\{x \in X: g(x)=n\} .
$$

Обозначим

$$
\hat{G}=\left\{\xi_{g}^{n}: g \in G, n \in \mathbf{N}\right\} .
$$

Определение понятия ИСП разбивается на два этапа. На первом этапе раскрывается структурная (схемная) часть этого понятия, на втором функциональная.

Дадим определение ИСП с точки зрения ее структуры. Пусть дана ориентированная многополюсная сеть. Выделим в ней один полюс и назовем его корнем, а остальные полюсы назовем листьями.

Выделим в сети некоторые вершины и назовем их точками переключения (полюсы могут быть точками переключения).

Если $\beta$ - вершина сети, то через $\psi_{\beta}$ обозначим полустепень исхода вершины $\beta$.

Каждой точке переключения $\beta$ сопоставим некий символ из $G$ такой, что максимальное значение переключателя, соответствующего этому символу, не превышает $\psi_{\beta}$. Это соответствие назовем нагрузкой точек переключения.

Для каждой точки переключения $\beta$ ребрам, из нее исходящим, поставим во взаимо однозначное соответствие числа из множества $\left\{1, \ldots, \psi_{\beta}\right\}$. Эти ребра назовем переключательными, а это соответствие нагрузкой переключательных ребер.

Ребра, не являющиеся переключательными, назовем предикатными. Каждому предикатному ребру сети сопоставим некоторый символ из множества $F$. Это соответствие назовем нагрузкой предикатных ребер.

Сопоставим каждому листу сети некоторую запись из множества $Y$. Это соответствие назовем нагрузкой листьев.

Полученную нагруженную сеть назовем информационной сетью с переключателями над базовым множеством $\mathscr{F}=\langle F, G\rangle$.

Дадим теперь определение функционирования ИСП. Пусть дана ИСП $U$. 
Последовательность ориентированных ребер сети

$$
\left(\alpha_{1}, \alpha_{2}\right),\left(\alpha_{2}, \alpha_{3}\right), \ldots,\left(\alpha_{m-1}, \alpha_{m}\right)
$$

назовем ориентированной цепью от вершины $\alpha_{1}$ к вершине $\alpha_{m}$.

Если $c$ ребро сети, то через $[c]$ обозначим его нагрузку.

Проводимостью ребра $(\alpha, \beta)$ пазовем предикат, равный $[(\alpha, \beta)]$, если ребро предикатное, и $\xi_{g}^{[(\alpha, \beta)]}$, если ребро переключательное, где $g$ - переключатель, соответствующий вершине $\alpha$.

Проводимостью ориентированной цепи назовем конъюнкцию проводимостей ребер цепи.

Если фиксировать запрос $x$, то цепь, проводимость которой на запросе $x$ равна 1 , назовем проводящей цепью на запросе $x$.

В ИСП по аналогии с контактными схемами введем для каждой пары вершин $\alpha$ и $\beta$ функцию проводимости $f_{\alpha \beta}$ от вершины $\alpha$ к вершине $\beta$ следующим образом:

если $\alpha=\beta$, то $\left.f_{\alpha \beta}(x)=1, x \in X\right)$;

если $\alpha \neq \beta$ и не существует в ИСП ориентированных цепей от вершины $\alpha$ к $\beta$, то $f_{\alpha \beta}(x)=0, x \in X$

если $\alpha \neq \beta$ и множество ориентированных цепей от $\alpha$ к $\beta$ не пусто, то $f_{\alpha \beta}(x)$ равно дизъюнкции проводимостей всех ориентированных цепей от $\alpha$ к $\beta$.

Функцию проводимости от корня ИСП к некоторой вершине $\beta$ ИСП назовем функцией фильтра вершины $\beta$ и обозначим $\varphi_{\beta}(x)$.

Через $\mathscr{R}(U), \mathscr{P}(U), \mathscr{L}(U)$ (или просто $\mathscr{R}, \mathscr{P}, \mathscr{L}$ ) обозначим множества вершін, точек переключения и листьев сети $U$ соответственно.

Пусть $\mathscr{N}$ - некоторая подсеть (то есть произвольное подмножество вершин и ребер) ИСП $U$. Через $\langle\mathcal{N}\rangle$ обозначим множество записей, соответствующих листьям этой подсети (в частности, если $\alpha-$ некоторый лист сети $U$, то под $\langle\alpha\rangle$ будем понимать запись, соответствующую листу $\alpha$ ).

Будем говорить, что ИСП $U$ реализует функцию $\mathscr{J}: X \rightarrow 2^{Y}$, называемую функцией ответа сети $U$ и определяемую соотношением

$$
\mathscr{J}(x)=\left\langle\left\{\alpha \in \mathscr{L}(U): \varphi_{\alpha}(x)=1\right\}\right\rangle .
$$

Понятие ИСП полностью определено.

В случае, когда базовое множество переключателей $G$ пусто, то есть в сетях нет переключателей, то ИСП называются информационными сетями с дублированием листьев (ИСД).

В данной статье мы будем в основном исследовать именно ИСД.

Скажем, что ИСП $U$ разрешает ЗИП $I=\langle X, V, \rho\rangle$, если для всех $x \in X$

$$
\mathscr{J}(x)=\{y \in V: x \rho y\}
$$

Сложностью ИСП $U$ на запросе $x$ назовем число

$$
T(U, x)=b \sum_{\beta \in \mathscr{P} \backslash \backslash \mathscr{P}} \psi_{\beta} \varphi_{\beta}(x)+a \sum_{\beta \in \mathscr{P}} \varphi_{\beta}(x),
$$

где константа $a$ характеризует сложность вычисления одного переключателя, а константа $b$ одного предиката. 
Далее всюду будем предполагать, что мы находимся в условиях леммы 1 из [4], то есть будем считать, что алгебра $\sigma$ содержит все множества $N_{f}$, где $f \in F \cup \hat{G}$.

При этих условиях $T(U, x)$ - случайная величина по $x$, и можно ввести понятия сложности сети и сложности ребра сети.

Сложностью ИСП $U$ назовем математическое ожидание величины $T(U, x)$, то есть число

$$
T(U)=\mathbf{M} T(U, x) .
$$

Если $(\beta, \alpha)$ - ребро ИСП, то сложностью этого ребра назовем число $b \mathbf{P}\left(N_{\varphi_{\beta}}\right)$, если $(\beta, \alpha)$ - предикатное ребро, и число $a \mathbf{P}\left(N_{\varphi_{\beta}}\right) / \psi_{\beta}$, если это ребро переключательное.

Легко видеть, что сложность ИСП равна сумме сложностей ребер ИСП, то есть

$$
T(U)=b \sum_{\beta \in \mathscr{R} \backslash \mathscr{P}} \psi_{\beta} \mathbf{P}\left(N_{\varphi_{\beta}}\right)+a \sum_{\beta \in \mathscr{P}} \mathbf{P}\left(N_{\varphi_{\beta}}\right)
$$

Далее всюду будем предполагать, что $a=b=1$.

Пусть дана некая ЗИП $I$. Сложностью задачи $I$ при базовом множестве $\mathscr{F}$ назовем число

$$
T(I, \mathscr{F})=\inf \{T(U): U \in \mathscr{U}(I, \mathscr{F})\}
$$

где $\mathscr{U}(I, \mathscr{F})$ - множество всех ИСП над базовым множеством $\mathscr{F}$, разрешающих ЗИП $I$.

Скажем, что базовое множество $\mathscr{F}$ полно для типа $S$, если $\mathscr{U}(I, \mathscr{F}) \neq \varnothing$ для любой ЗИП $I$ типа $S$.

Пусть $M$ - некоторое множество. Через $|M|$ обозначим число элементов в множестве $M$, называемое мощностью множества $M$.

Как обычно, будем писать $\alpha(n)=o(1)$, если $\alpha(n) \rightarrow 0$ при $n \rightarrow \infty$, и $A(n)=$ $\circ(B(n))$, если $A(n)=B(n) o(1)$.

Скажем, что $A(n)$ асимптотически не превосходит $B(n)$ при $n \rightarrow \infty$ и обозначим $A \lesssim B$, если существует такое $\alpha(n)=o(1)$, что $A(n) \leqslant(1+\alpha(n)) B(n)$, начиная с некоторого $n_{0}$.

Если $A \lesssim B$ и $B \lesssim A$, то будем говорить, что $A$ и $B$ асимптотически равны при $n \rightarrow \infty$ и писать в этом случае $A \sim B$.

Через $\left(\begin{array}{l}n \\ k\end{array}\right)$ будем обсзначать число сочетаний из $n$ элементов по $k$.

Рассмотрим задачи поиска типа

$$
S_{b}=\left\langle B^{n}, B^{n}, \succeq, \sigma, \mathbf{P}\right\rangle,
$$

где $B^{n}-$ единичный $n$-мерный куб, то есть

$$
B^{n}=\left\{\left(\alpha_{1}, \ldots, \alpha_{n}\right): \alpha_{i} \in\{0,1\}, i=1, \ldots, n\right\}
$$

$\succeq$ - отношение поиска на $B^{n} \times B^{n}$, определяемое соотношением

$$
\left(x_{1}, \ldots, x_{n}\right) \succeq\left(y_{1}, \ldots, y_{n}\right) \Longleftrightarrow x_{i} \geqslant y_{i}, \quad i=1, \ldots, n,
$$

$\sigma$ - алгебра подмножеств $B^{n}$, представляющая собой множество всех подмножеств $B^{n}, \mathbf{P}$ - равномерная вероятностная мера на $\sigma$, то есть такая мера, что $\mathbf{P}(x)=1 / 2^{n}$ для всех $x \in B^{n}$ и $\mathbf{P}(A)=|A| / 2^{n}$ для всех $A \subseteq B^{n}$. 
Задачи поиска, принадлежащие данному типу, суть разновидность задач, именуемых в литературе задачами включающего поиска (см., например, [5]), поэтому тип $S_{b}$ мы назовем типом включающего поиска, а задачи, принадлежащие этому типу, задачами включающего поиска.

Напомним, что в соответствии с терминологией [6] весом набора $\left(\alpha_{1, \ldots}, \ldots, \alpha_{n}\right) \in$ $B^{n}$ называют число его координат, равных единице. Формула

$$
x_{i_{1}}^{\sigma_{1}} \& x_{i_{2}}^{\sigma_{2}} \& \ldots \& x_{i_{r}}^{\sigma_{r}}
$$

где $\&$ - знак конъюнкции, $\sigma_{k} \in\{0,1\}, x_{i_{k}}^{0}=\bar{x}_{i_{k}}, x_{i_{k}}^{1}=x_{i_{k}}, i_{k} \in\{1,2, \ldots, n\}$, $k=1,2, \ldots, r(r \geqslant 1$ и $n \geqslant 1)$, называется конъюнкцией над множеством переменных $X^{n}=\left\{x_{1}, x_{2}, \ldots, x_{n}\right\}$. Если $x_{i_{j}} \neq x_{i_{k}}$ при $j \neq k$, то конъюнкция называется элементарной. Элементарная конъюнкция называется монотонной, если она не содержит отрицаний переменных. Множество элементарных монотонных конъюнкций от $n$ переменных будем обозначать через $\mathscr{K}^{n}$.

Рассмотрим произвольную запись $y \in B^{n}$. Пусть $\left\{i_{1}, \ldots, i_{k}\right\}$ есть множество номеров координат вектора $y$, которые равны единице. Нетрудно видеть, что

$$
\chi_{y, \succeq}\left(x_{1}, \ldots, x_{n}\right)=x_{i_{1}} \& x_{i_{2}} \& \ldots \& x_{i_{k}},
$$

то есть характеристическая функция записи $y$ является элементарной монотонной конъюнкцией.

Отсюда согласно теореме 1 из [4] сеть, разрешающая некоторую задачу включающего поиска, представляет собой сеть, реализующую некоторую систему элементарных монотонных конъюнкций. Откуда согласно теореме 2 из [1] базовое множество $\left\langle X^{n}, \varnothing\right\rangle$ является полным для типа $S_{b}$.

Справедлива следуюшая теорема.

Теорема 1. Если базовое множество имеет вид $\mathscr{F}=\left\langle X^{n}, \varnothing\right\rangle$ u $k(n), m(n) u \alpha(n)$ - такие числа, зависящие от $n$, что

$$
\begin{gathered}
k(n) \sim\left(\begin{array}{c}
n \\
m-1
\end{array}\right) \alpha(n), \quad m(n) \geqslant 1, \quad m(n)=o(\log n / \log \log n), \\
\alpha(n) \rightarrow \infty, \quad(\log n-\log \alpha(n)) /(m(n) \log (m(n)+1)) \rightarrow \infty
\end{gathered}
$$

nри $n \rightarrow \infty$, то для любого такого $k(n)$ существуют такие ЗИП $I_{k}=\left\langle B^{n}, V_{k}, \succeq\right\rangle$ muпа $S_{b}$, которых $\left|V_{k}\right|=k$, что для них при $n \rightarrow \infty$ справедливо соотношение

$$
\sum_{y \in V} \mathbf{P}(O(y, \succeq))=o\left(\inf \left\{T(U): U \in \mathscr{U}_{d}(I, \mathscr{F})\right\}\right),
$$

где $\mathscr{U}_{d}(I, \mathscr{F})$ - множество всех ИСД над базовым множеством $\mathscr{F}$, разрешающих ЗИП І и граф которых имеет вид дерева.

\section{3. Доказательство теоремы}

Приведем доказательство теоремы 1.

Пусть $p=p(n)$ - такое целое число, что $p^{m(n)} \geqslant k=k(n)>(p-1)^{m(n)}$. Оценим величину $p / n$. Для этого рассмотрим два случая, когда $m=m(n)$ фиксировано и когда $m \rightarrow \infty$ при $n \rightarrow \infty$. 
В первом случае

$$
\begin{aligned}
\frac{p}{n} & \sim \frac{k^{1 / m}}{n} \sim n^{-1}\left(\frac{\alpha n(n-1) \cdots(n-m+2)}{(m-1) !}\right)^{1 / m} \\
& \sim\left(\frac{n^{m-1} \alpha}{n^{m}(m-1) !}\right)^{1 / m}=\left(\frac{\alpha}{n(m-1) !}\right)^{1 / m}=o(1)
\end{aligned}
$$

так как, как легко видеть, $\alpha(n)=o(n)$.

Во втором случае, используя формулу Стирлинга (см., например, [6]), получаем аналогичный результат

$$
\begin{aligned}
\frac{p}{n} & \sim \frac{k^{1 / m}}{n} \sim n^{-1}\left(\frac{\alpha}{\sqrt{2 \pi(m-1)}}\left(\frac{n e}{m-1}\right)^{m-1}\right)^{1 / m} \\
& =\left(\frac{n^{m-1} \alpha}{n^{m}}\right)^{1 / m}\left(\frac{e}{m-1}\right)^{m /(m-1)}(2 \pi(m-1))^{-1 / 2 m}=o(1) .
\end{aligned}
$$

Пусть $r=r(n)$ таково, что

$$
p m+p \sum_{i=1}^{r} m^{i} \leqslant n
$$

Договоримся представлять запись $y \in B^{n}$ ее характеристической функцией, то есть представлять ее в виде конъюнкции переменных, номера которых совпадают с номерами разрядов вектора $y$, в которых стоят единицы.

Разобьем все множество переменных $X^{n}$ на $m+r+1$ взаимно не пересекающиеся части так, что в первых $m$ частях находится по $p$ переменных, в $(m+i)$-и части $(i=1, \ldots, r)$ находится $p m^{i}$ переменных, а в $(m+r+1)$-й части все оставшиеся переменные. В соответствии с неравенством (3) такое разбиение возможно. Переменные из $i$-й части $X^{n}$ при $i=1, \ldots, m+r+1$ будем обозначать через $x_{q}^{i}$, то есть верхний индекс переменной будет говорить, какой части принадлежит переменная.

Рассмотрим библиотеку

$$
V=\left\{x_{q_{1}}^{1} x_{q_{2}}^{2} \ldots x_{q_{m}}^{m} x_{f_{1}}^{m+1} \ldots x_{f_{r}}^{m+r}: q_{j} \in\{1, \ldots, p\}, j=1, \ldots, m\right\},
$$

где

$$
f_{j}=f_{j}\left(q_{1}, \ldots, q_{m}\right)=\sum_{i=1}^{m} q_{i} i^{j-1}, \quad j=1, \ldots, r .
$$

Поскольку каждая запись

$$
x_{q_{1}}^{1} x_{q_{2}}^{2} \ldots x_{q_{m}}^{m} x_{f_{1}}^{m+1} \ldots x_{f_{r}}^{m+r}
$$

полностью определяется набором $\left(q_{1}, \ldots, q_{m}\right)$, мощность библиотеки $V$ равна $|V|=$ $p^{m} \geqslant k$. Отметим также, что для любого $j=1, \ldots, r$

$$
f_{j}=\sum_{i=1}^{m} q_{i} i^{j-1} \leqslant p m m^{j-1}=p m^{j} .
$$


Покажем, что любая пара различных векторов из $V$ имеет не более чем $m-1$ одинаковых переменных. Возьмем произвольную пару векторов из $V$

$$
\begin{gathered}
x_{q_{1}}^{1} x_{q_{2}}^{2} \ldots x_{q_{m}}^{m} x_{f_{1}}^{m+1} \ldots x_{f_{r}}^{m+r} \\
x_{q_{1}^{\prime}}^{1} x_{q_{2}^{\prime}}^{2} \ldots x_{q_{m}^{\prime}}^{m} x_{f_{1}^{\prime}}^{m+1} \ldots x_{f_{r}^{\prime}}^{m+r} .
\end{gathered}
$$

Пусть $q_{i_{l}} \neq q_{i_{l}}^{\prime}$, при $i_{l} \in\{1, \ldots, m\}, l=1, \ldots, s$, и $q_{j}=q_{j}^{\prime}$, если $j$ принадлежит $\{1, \ldots, m\} \backslash\left\{i_{l}: l=1, s\right\}$. Так как векторы различны, $s>0$.

Пусть $f_{j_{l}}=f_{j_{l}}^{\prime}$ при $j_{l} \in\{1, \ldots, r\}, l=1, \ldots, t$, и $f_{j} \neq f_{j}^{\prime}$, если $j$ принадлежит $\{1, \ldots, r\} \backslash\left\{j_{l}: l=1, \ldots, t\right\}$. Покажем, что $t<s$.

Предположим, что это не так, то есть $t \geqslant s$.

Рассмотрим систему уравненй

$$
\begin{gathered}
f_{j_{1}}=f_{j_{1}}^{\prime}, \\
\cdots \\
f_{j_{t}}=f_{j_{t}}^{\prime} .
\end{gathered}
$$

Взяв в этой системе первые $s$ уравнений, получим систему

$$
\begin{gathered}
\sum_{i=1}^{m} q_{i} i^{j_{1}-1}=\sum_{i=1}^{m} q_{i}^{\prime} i^{j_{1}-1}, \\
\sum_{i=1}^{m} q_{i} i^{j_{s}-1}=\sum_{i=1}^{m} q_{i}^{\prime} i^{j_{s}-1},
\end{gathered}
$$

откуда

$$
\begin{gathered}
\sum_{l=1}^{s}\left(q_{i_{l}}-q_{i_{l}}^{\prime}\right) i_{l}^{j_{1}-1}=0 \\
\cdots \\
\sum_{l=1}^{s}\left(q_{i_{l}}-q_{i_{l}}^{\prime}\right) i_{l}^{j_{s}-1}=0
\end{gathered}
$$

Определитель этой системы

$$
\left|\begin{array}{cccc}
i_{1}{ }^{j_{1}-1} & i_{2}{ }^{j_{1}-1} & \ldots & i_{s}{ }^{j_{1}-1} \\
i_{1}{ }^{j_{2}-1} & i_{2}{ }^{j_{2}-1} & \ldots & i_{s}{ }^{j_{2}-1} \\
& & \ldots & \\
i_{1}{ }^{j_{s}-1} & i_{2}{ }^{j_{s}-1} & \ldots & i_{s}{ }^{j_{s}-1}
\end{array}\right|
$$

есть минор определителя Вандермонда и не равен нулю, поскольку $i_{1}, i_{2}, \ldots, i_{s}$ - положительные числа. Этот простой факт приведен в качестве упражнения, например, в [7]. Поэтому эта система имеет единственное решение

$$
\begin{gathered}
q_{i_{1}}=q_{i_{1}}^{\prime}, \\
\ldots \\
q_{i_{s}}=q_{i_{s}}^{\prime} .
\end{gathered}
$$


Но согласно предположению

$$
\begin{aligned}
q_{i_{1}} & \neq q_{i_{1}}^{\prime}, \\
& \ldots \\
q_{i_{s}} & \neq q_{i_{s}}^{\prime} .
\end{aligned}
$$

Полученное противоречие доказывает, что $t<s$ и, следовательно, любая пара записей из $V$ имеет не более, чем $m-1$ общих переменных.

Рассмотрим такую библиотеку $V^{\prime}$, что $V^{\prime} \subset V,\left|V^{\prime}\right|=k$. Пусть $I=\left\langle B^{n}, V^{\prime}, \succeq\right\rangle$ - соответствующая этой библиотеке ЗИП типа $S_{b}$. Пусть $U^{\prime}-$ произвольная древовидная сеть из $\mathscr{U}_{d}(I, \mathscr{F})$.

Возьмем произвольную запись $y \in V^{\prime}$. Главной цепью записи $y$ назовем цепь, ведущую из корня в какой-либо лист, которому приписана запись $y$, такую, что проводимость этой цепи равна характеристической функции записи $y$. Как показано в [2], для любой записи $y$ из $V^{\prime}$ в сети $U^{\prime}$ существует главная цепь, которую обозначим $C_{y}$. Для каждой записи из $V^{\prime}$ выберем по главной цепи и далее будем говорить только об этих главных цепях. Длина цепи $C_{y}$ не меньше, чем $m+r$, и каждому ребру этой цепи приписана некоторая переменная из характеристической функции записи $y$. Пусть $y=x_{i_{1}} x_{i_{2}} \ldots x_{i_{m+r}}$, где переменные упорядочены в порядке следования вдоль цепи $C_{y}$ (если некоторая переменная встречается в цепи дважды и более, то упорядочиваем ее по первому вхождению). Сопоставим каждой переменной $x_{i_{j}}, j=1, \ldots, m+r$, первое ребро, которому приписана эта переменная. Сложность этого ребра не меньше, чем

$$
\mathbf{P}\left(N_{x_{i_{1}} \& x_{i_{2}} \& \ldots \& x_{i_{j-1}}}\right)=2^{1-j}
$$

Если ребро цепи $C_{y}$, соответствующее переменной $x_{i_{j}}$, принадлежит также некоторой другой главной цепи $C_{y^{\prime}}$, то все ребра, соответствующие переменным $x_{i_{1}}, \ldots, x_{i_{j-1}}$, также принадлежат цепи $C_{y^{\prime}}$, так как $U^{\prime}-$ древовидная сеть. Отсюда, учитывая, что любая пара записей из $V^{\prime}$ имеет не более, чем $m-1$ общих переменных, получаем, что все ребра, соответствующие переменным $x_{i_{m}}, \ldots, x_{i_{m+r}}$, принадлежат только одной главной цепи $C_{y}$. Таким образом, каждой записи из $V^{\prime}$ мы можем сопоставить цепочку из $r+1$ ребра, причем цепочки, соответствующие различным записям, не пересекаются. Суммарная сложность каждой такой цепочки не меньше, чем

$$
\sum_{j=0}^{r} 2^{1-m-j}=2^{2-m}\left(1-2^{-r-1}\right)
$$

Следовательно,

$$
T\left(U^{\prime}\right) \geqslant k 2^{2-m}\left(1-2^{-r-1}\right) .
$$

Возьмем $r=r(n)=\left[\log _{m+1}(n / p)\right]$. При таком выборе $r$

$$
p m+p \sum_{i=1}^{r} m^{i} \leqslant p(m+1)^{r} \leqslant n,
$$

то есть выполняется условие (3).

Оценим величину $r(n)$. Для этого, как и ранее, рассмотрим два случая, когда $m$ фиксировано, и когда $m \rightarrow \infty$ при $n \rightarrow \infty$. 
В первом случае, используя (1), получим, что

$$
r \geqslant \frac{\log (n / p)}{\log (m+1)}-1 \sim \frac{\log n-\log \alpha+\log ((m-1) !)}{m \log (m+1)},
$$

где правая часть стремится к бесконечности.

Во втором случае, используя (2), получим аналогичный результат:

$$
\begin{aligned}
r \geqslant & \frac{\log (n / p)}{\log (m+1)}-1 \sim \frac{1}{\log (m+1)}\left(\log n-\frac{m-1}{m} \log n-\frac{m-1}{m} \log e\right. \\
& \left.\quad+\frac{m-1}{m} \log (m-1)+\frac{1}{2 m} \log (2 \pi(m-1))-\frac{1}{m} \log \alpha\right) \\
= & \frac{\log n-\log \alpha+(m-1) \log ((m-1) / l)+\log \sqrt{2 \pi(m-1)}}{m \log (m+1)},
\end{aligned}
$$

где правая часть стремится к бесконечности.

Отсюда следует, что для любой сети $U^{\prime} \in \mathscr{U}_{d}(I, \mathscr{F})$

$$
T\left(U^{\prime}\right) \gtrsim k 2^{2-m} \text {. }
$$

Следовательно,

$$
\inf \left\{T(U): U \in \mathscr{U}_{d}(I, \mathscr{F})\right\} \gtrsim k 2^{2-m}
$$

Построим некоторую ИСД, которая разрешала бы задачу $I$ по аналогии с тем, как это делалось в теореме 3 из [2].

Обозначим через $\mathscr{K}_{l}^{n}$ множество всех монотонных элементарных конъюнкций, содержащих не более $l$ переменных (или другими словами, имеющих длину не более чем $l$ ).

Построим по индукции информационную сеть $U_{l}^{n}$, реализующую как функции фильтров каких-либо вершин все функции из $\mathscr{K}_{i}^{n}$.

В качестве базы индукции возьмем $l=1, \mathscr{K}_{1}^{n}=\left\{1, x_{1}, \ldots, x_{n}\right\}$.

Универсальный многополюсник $U_{1}^{n}$ для множества $\mathscr{K}_{1}^{n}$ будет иметь вид звезды, центр которой есть корень сети, а на концах ребер реализуются функции из $\mathscr{K}_{1}^{n}$.

Рассмотрим шаг индукции. Пусть $U_{l-1}^{n}-$ сеть, реализующая все функции из $\mathscr{K}_{l-1}^{n}$. Сеть $U_{l}^{n}$ будем строить, добавляя к $U_{l-1}^{n}$ вершины и ребра, чтобы реализовать функции из $\mathscr{K}_{l}^{n} \backslash \mathscr{K}_{l-1}^{n}$, следующим образом. Возьмем произвольную монотонную элементарную конъюнкцию $x_{i_{1}} \& \ldots \& x_{i_{l}}$ длины $l$. В сети $U_{l-1}^{n}$ найдем вершину, на которой реализуется функция $x_{i_{1}} \& \ldots \& x_{i_{l-1}}$ и выпустим из нее ребро, которому припишем переменную $x_{i_{l}}$. На вершине, в которую ведет это ребро, будет реализовываться как функция фильтра функция $x_{i_{1}} \& \ldots \& x_{i_{l}}$. Перебрав все функции из $\mathscr{K}_{l}^{n} \backslash \mathscr{K}_{l-1}^{n}$ и проделав для каждой эту операцию, получим требуемую сеть $U_{l}^{n}$, реализующую все функции из $\mathscr{K}_{l}^{n}$.

Отметим, что на первом ярусе сети $U_{l}^{n}$ находится

$$
n+1=\left(\begin{array}{l}
n \\
1
\end{array}\right)+1
$$

ребер, и сложность каждого из них равна единице (первый ярус - это ребра, исходящие из корня), а на $i$-ом ярусе, $i \geqslant 2$, находится $\left(\begin{array}{c}n \\ i\end{array}\right)$ ребер, и сложность каждого 
из них равна $2^{1-i}(i$-й ярус - это ребра, исходящие из концов ребер $(i-1)$-го яруса). Таким образом, сложность сети $U_{l}^{n}$ равна

$$
T\left(U_{l}^{n}\right)=1+\sum_{i=1}^{l}\left(\begin{array}{c}
n \\
i
\end{array}\right) 2^{1-i}
$$

Вернемся теперь к задаче $I$.

Информационную сеть $U$, разрешающую задачу I, будем строить следующим образом. Возьмем сеть $U_{m-1}^{n}$, описанную выше. Возьмем произвольную запись $y \in V$. Пусть она равна $x_{i_{1}} x_{i_{2}} \ldots x_{i_{m+r}}$. Возьілем в сети $U_{m-1}^{n}$ вершину, на которой реализуется конъюнкция $x_{i_{1}} x_{i_{2}} \ldots x_{i_{m-1}}$ и выпустим из нее цепочку ребер, которым приписаны переменные $x_{i_{m}}, x_{i_{m+1}}, \ldots, x_{i_{m+r}}$. И такую операцию проделаем для каждой записи $y \in V$. Понятно, что полученная сеть $U$ над базовым множеством $\mathscr{F}$ будет разрешать задачу $I$.

Подсчитаем сложность сети $U$. Она состоит из сети $U_{m-1}^{n}$ и $k$ цепочек, каждая из которых содержит $r+1$ ребер, и сложность которой равна, как мы отмечали выше, $2^{2-m}\left(1-2^{-r-1}\right)$, поэтому

$$
T(U)=1+\sum_{i=1}^{m-1} 2^{1-i}\left(\begin{array}{l}
n \\
i
\end{array}\right)+2^{2-m}\left(1-2^{-r-1}\right)=k 2^{2-m}(1+o(1)) .
$$

Отсюда, учитывая неравенство (4), получаем, что

$$
\inf \left\{T(U): U \in \mathscr{U}_{d}(I, \mathscr{F})\right\}=k 2^{2-m}(1+o(1)) .
$$

Для завершения доказательства теоремы осталось заметить, что

$$
\frac{1}{k 2^{2-m}} \sum_{y \in V^{\prime}} \mathbf{P}(O(y, \succeq))=k 2^{-m-r} /\left(k 2^{2-m}\right)=2^{-2-r}=o(1) .
$$

Тем самым, теорема доказана.

\section{Список литературы}

1. Гасанов Э. Э. Об одной математической модели информационного поиска. Дискретная математиха (1991) 3, №2, 69-76.

2. Гасанов Э. Э. Нижняя оценка сложности информационных сетей для одного отношения частичного порядка. Дискретная математиха (1996) 8, №4, 108-122.

3. Гасанов Э. Э., Косолапов А. В. К вопросу о древовидности оптимальных информационных сетени включающего поиска. Интеллектуальные системы (1997) 2, ???

4. Гасанов Э. Э. Об одномерной задаче интервального поиска. Дискретная математика (1995) 7, №2, 40-60.

5. Селтон Г. Аөтоматическая обработка, хранение и поиск информачии. Сов. радио, Москва, 1973.

6. Гаврилов Г. П., Сапоженко А. А. Задачи и упражнения по курсу дискретной математики. Наука, Москва, 1992.

7. Полиа Г., Сегё Г. Задачи и теоремы из анализа. Наука, Москва, 1978.

Статья поступила 13.10.1997. 